\title{
Tests of the Equivalence Principle with neutral kaons
}

\section{CPLEAR Collaboration}

A. Apostolakis ${ }^{\text {a }}$, E. Aslanides ${ }^{\text {k }}$, G. Backenstoss ${ }^{\text {b }}$, P. Bargassa ${ }^{\text {m }}$, O. Behnke ${ }^{\mathrm{q}}$, A. Benelli ${ }^{\mathrm{b}}$, V. Bertin ${ }^{\mathrm{k}}$, F. Blanc ${ }^{\mathrm{g}, \mathrm{m}}$, P. Bloch ${ }^{\mathrm{d}}$, P. Carlson ${ }^{\circ}, \mathrm{M}$. Carroll ${ }^{\mathrm{i}}$, E. Cawley ${ }^{\text {i }, ~ G . ~ C h a r d i n ~}{ }^{\text {n }}$, M.B. Chertok ${ }^{\mathrm{c}}$, M. Danielsson ${ }^{\circ}$, M. Dejardin ${ }^{\mathrm{n}}$, J. Derre ${ }^{\mathrm{n}}$, A. Ealet ${ }^{\mathrm{k}}$, C. Eleftheriadis ${ }^{\mathrm{p}}$, L. Faravel ${ }^{\mathrm{g}}$, W. Fetscher ${ }^{\mathrm{q}}$, M. Fidecaro ${ }^{\mathrm{d}}$, A. Filipčič ${ }^{j}$, D. Francis ${ }^{c}$, J. Fry ${ }^{\text {i }}$, E. Gabathuler ${ }^{\text {i }}$, R. Gamet ${ }^{i}$, H.-J. Gerber ${ }^{q}$, A. Go ${ }^{\text {d }}$, A. Haselden ${ }^{\text {i }}$, P.J. Hayman ${ }^{\text {i }}$, F. Henry-Couannier ${ }^{k}$, R.W. Hollander ${ }^{\text {f }}$, K. Jon-And ${ }^{\circ}$, P.-R. Kettle ${ }^{\mathrm{m}}$, P. Kokkas ${ }^{\mathrm{d}}$, R. Kreuger ${ }^{\mathrm{f}}$, R. Le Gac ${ }^{\mathrm{k}}$, F. Leimgruber ${ }^{\text {b }}$, I. Mandić ${ }^{\mathrm{j}}$, N. Manthos ${ }^{\mathrm{h}}$, G. Marel ${ }^{\mathrm{n}}$, M. Mikuž ${ }^{\mathrm{j}}$, J. Miller ${ }^{\mathrm{c}}$, F. Montanet ${ }^{\mathrm{k}}$, A. Muller ${ }^{\mathrm{n}}$, T. Nakada ${ }^{\mathrm{m}}$, B. Pagels ${ }^{\mathrm{q}}$, I. Papadopoulos ${ }^{\mathrm{p}}$, P. Pavlopoulos ${ }^{\text {b }}$, G. Polivka ${ }^{\text {b }}$, R. Rickenbach ${ }^{\text {b }}$, B.L. Roberts ${ }^{\text {c }}$, T. Ruf ${ }^{\text {d, }}$, L. Sakeliou ${ }^{\text {a }}$, M. Schäfer ${ }^{\text {q }}$, L.A. Schaller ${ }^{\mathrm{g}}$, T. Schietinger ${ }^{\mathrm{b}}$, A. Schopper ${ }^{\mathrm{d}}$, L. Tauscher ${ }^{\text {b }}$, C. Thibault ${ }^{1}$, F. Touchard ${ }^{k}$, C. Touramanis i , C.W.E. Van Eijk ${ }^{\text {f }}$, S. Vlachos ${ }^{\text {b }}$, P. Weber ${ }^{\mathrm{q}}$, O. Wigger ${ }^{\mathrm{m}}$, M. Wolter ${ }^{\mathrm{q}}$, D. Zavrtanik ${ }^{\mathrm{j}}$, D. Zimmerman ${ }^{\text {c }}$, John Ellis ${ }^{\mathrm{r}}$, N.E. Mavromatos ${ }^{\mathrm{s}}$, D.V. Nanopoulos ${ }^{\mathrm{t}}$

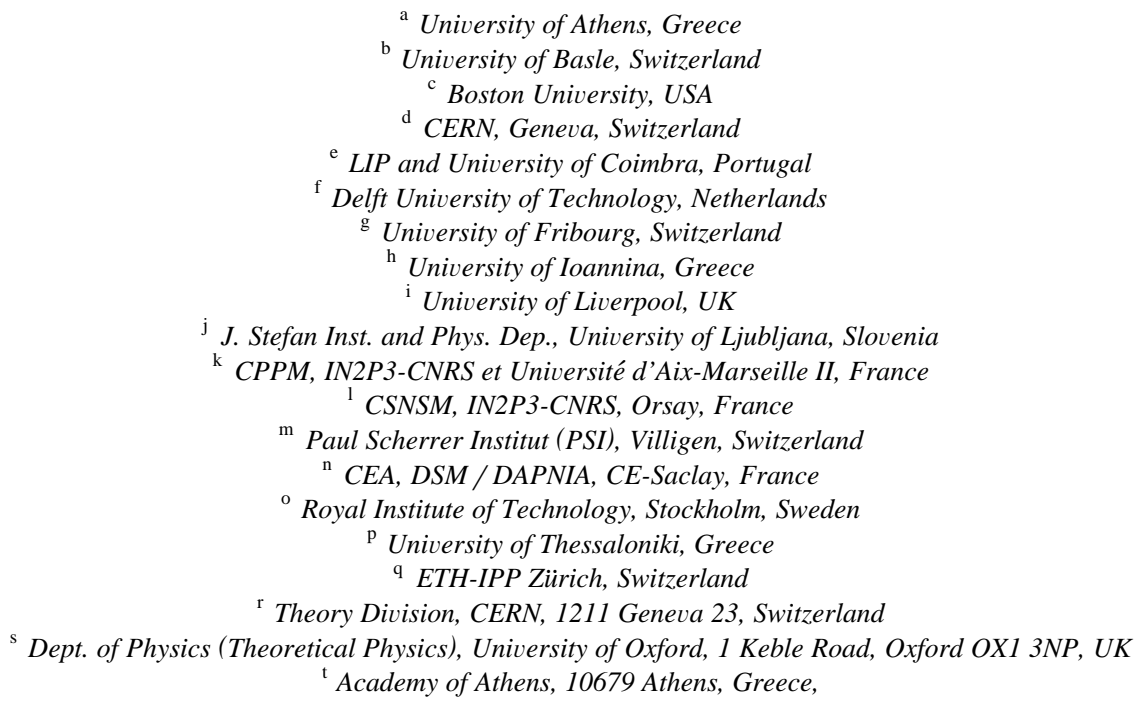

${ }^{a}$ University of Athens, Greece

${ }^{\mathrm{b}}$ University of Basle, Switzerland

${ }^{\mathrm{c}}$ Boston University, USA

d CERN, Geneva, Switzerland

${ }^{\mathrm{e}}$ LIP and University of Coimbra, Portugal

${ }^{\mathrm{f}}$ Delft University of Technology, Netherlands

${ }^{\mathrm{g}}$ University of Fribourg, Switzerland

${ }^{\mathrm{h}}$ University of Ioannina, Greece

${ }^{\mathrm{i}}$ University of Liverpool, $U K$

${ }^{j}$ J. Stefan Inst. and Phys. Dep., University of Ljubljana, Slovenia

${ }^{\mathrm{k}}$ CPPM, IN2P3-CNRS et Université d'Aix-Marseille II, France

${ }^{1}$ CSNSM, IN2P3-CNRS, Orsay, France

${ }^{\mathrm{m}}$ Paul Scherrer Institut (PSI), Villigen, Switzerland

${ }^{n}$ CEA, DSM / DAPNIA, CE-Saclay, France

${ }^{\circ}$ Royal Institute of Technology, Stockholm, Sweden

${ }^{\mathrm{p}}$ University of Thessaloniki, Greece

${ }^{\mathrm{q}}$ ETH-IPP Zürich, Switzerland

${ }^{\mathrm{r}}$ Theory Division, CERN, 1211 Geneva 23, Switzerland

s Dept. of Physics (Theoretical Physics), University of Oxford, 1 Keble Road, Oxford OX1 3NP, UK

${ }^{\mathrm{t}}$ Academy of Athens, 10679 Athens, Greece, 
Center for Theoretical Physics, Department of Physics, Texas A\& M University, College Station, TX 77843-4242, USA, and Astroparticle Physics Group, Houston Advanced Research Center, The Mitchell Campus, The Woodlands, TX 77381, USA

Received 21 December 1998; received in revised form 22 February 1999

Editor: L. Montanet

\begin{abstract}
We test the Principle of Equivalence for particles and antiparticles, using CPLEAR data on tagged $\mathrm{K}^{0}$ and $\overline{\mathrm{K}}^{0}$ decays into $\pi^{+} \pi^{-}$. For the first time, we search for possible annual, monthly and diurnal modulations of the observables $\left|\eta_{+-}\right|$and $\phi_{+-}$, that could be correlated with variations in astrophysical potentials. Within the accuracy of CPLEAR, the measured values of $\left|\eta_{+-}\right|$and $\phi_{+-}$are found not to be correlated with changes of the gravitational potential. We analyze data assuming effective scalar, vector and tensor interactions, and we conclude that the Principle of Equivalence between particles and antiparticles holds to a level of $6.5,4.3$ and $1.8 \times 10^{-9}$, respectively, for scalar, vector and tensor potentials originating from the Sun with a range much greater than the distance Earth-Sun. We also study energy-dependent effects that might arise from vector or tensor interactions. Finally, we compile upper limits on the gravitational coupling difference between $\mathrm{K}^{0}$ and $\overline{\mathrm{K}}^{0}$ as a function of the scalar, vector and tensor interaction range. (C) 1999 Elsevier Science B.V. All rights reserved.
\end{abstract}

\section{Introduction}

The neutral kaon system is a very sensitive laboratory for the exploration of possible differences between matter and antimatter. Indeed, it is the only system where any matter-antimatter difference has been seen, which is conventionally ascribed to a CP-violating term in the $\mathrm{K}^{0}-\overline{\mathrm{K}}^{0}$ mass matrix. Searches for other asymmetries between $\mathrm{K}^{0}$ and $\overline{\mathrm{K}}^{0}$ have also been conducted, notably to set upper limits on effects violating CPT invariance [1]. These have included a possible [2] CPT-violating $\mathrm{K}^{0}-\overline{\mathrm{K}}^{0}$ mass difference, $\delta m$, and width difference, $\delta \Gamma,[3,4]$ and stochastic CPT violation of the form that may appear if neutral kaons should be described as an open quantum-mechanical system [5-7]. These searches have been motivated in part by suggestions that some form of CPT violation may occur in a quantum theory of gravity [8].

It has also been suggested that some quantum theory of gravity might entail an apparent violation of the Principle of Equivalence between matter and antimatter particles, due for instance to the possible exchange of a light vector particle with interactions of gravitational strength, a "graviphoton", [9]. Such a deviation from the Principle of Equivalence would imply a different gravitational coupling between particle and antiparticle, independently of the universality of the coupling between matter and gravity [10].
The aspect of the Principle of Equivalence concerning the universality of the coupling between the graviton and matter has been verified by many and diverse experiments, which, over the last four decades, have placed stringent limits on possible deviations from General Relativity. These include phenomenological searches for non-universality in free fall [11], the fractional difference in the acceleration of the Earth and Moon in the Sun's gravitational field using lunar laser ranging [12], the universality of the gravitational red shift using very stable clocks on aircraft, rockets and satellites [13], the spatial anisotropy of nuclear energy levels [14], and upper limits on time variations in the basic coupling constants of the Standard Model [15].

The other aspect of the Equivalence Principle, that of the universality of the gravitational coupling between matter and antimatter, can be tested by particle-antiparticle comparisons, such as $\mathrm{K}^{0}-\overline{\mathrm{K}}^{0}$ [4] and $p-\bar{p}$ [16] mass-difference measurements, which can be interpreted as tests of the Principle of Equivalence under the assumption of exact CPT symmetry [17].

Data from the CPLEAR experiment have been used previously to set the most precise available upper limits on a possible CPT-violating $\mathrm{K}^{0}-\overline{\mathrm{K}}^{0}$ mass difference, $\delta m$, and width difference, $\delta \Gamma$, [4] and on stochastic CPT-violating parameters [7]. These data are used in the present paper as a function of 
time, in order to provide tests of the second aspect of the Principle of Equivalence comparing $\mathrm{K}^{0}$ and $\overline{\mathrm{K}}^{0}$. After presenting the formalism, we report on a systematic search for possible annual, monthly and diurnal modulations of the observables $\left|\eta_{+-}\right|$and $\phi_{+-}$, as functions of the known variations of the astrophysical potentials. The upper limits so obtained can be used to constrain differences between $\mathrm{K}^{0}$ and $\overline{\mathrm{K}}^{0}$ interactions with a background field, as a function of its intrinsic spin and range. We use these bounds to discuss the possibility that all of the observed $\mathrm{CP}$ violating effects could be due to an astrophysical scalar field [18]. In addition, the $\mathrm{K}^{0}-\overline{\mathrm{K}}^{0}$ mass difference provided by the CPLEAR experiment, which is ten orders of magnitude more precise than the available $\mathrm{p}-\overline{\mathrm{p}}$ mass difference, puts stronger upper limits on the violation of the Principle of Equivalence for longer-range interactions, by considering galactic and extragalactic background fields. Finally, we comment on the possible combination of CPLEAR data with higher-energy data to constrain more tightly interactions with vector or tensor background fields.

Although limits on differences between $\mathrm{K}^{0}$ and $\overline{\mathrm{K}}^{0}$ interactions with a background field, arising from limits on the $\mathrm{K}^{0}-\overline{\mathrm{K}}^{0}$ mass difference, have already been reported in the literature [19], limits derived from studies of the observables $\left|\eta_{+-}\right|$and $\phi_{+-}$, in relation with the known time variations of the astrophysical potentials, are, to our knowledge, entirely new. We note that limits derived from possible modulations of $\left|\eta_{+-}\right|$and $\phi_{+-}$related to the modulation of astrophysical potentials do not depend on the less well known galactic and extragalactic potentials, those being constant during the lifetime of the experiment.

\section{Formalism for equivalence tests}

The standard treatment of mass mixing in the neutral kaon system is based on the following parameterization

$$
\mathscr{M}=\left(\begin{array}{lr}
M_{\mathrm{K}^{0}} & \Delta m / 2 \\
\Delta m / 2 & M_{\mathrm{K}^{0}}
\end{array}\right)
$$

where $M_{\mathrm{K}^{0}}$ is the kaon inertial mass, and $\Delta m=m_{\mathrm{L}}$ $-m_{\mathrm{S}}$ is the mass difference between the long- and short-lived neutral kaons. Non-invariance under CPT would induce, in principle, a mass difference, $\delta m$, between the $\mathrm{K}^{0}$ and $\overline{\mathrm{K}}^{0}$, which is limited to $\delta m \leq$ $3.5 \times 10^{-19} \mathrm{GeV}$ (95\% CL) [4]. We assume in (1) and the rest of this paper the equality of the inertial masses and widths of $\mathrm{K}^{0}$ and $\overline{\mathrm{K}}^{0}$. Also, we work throughout in the conventional quantum-mechanical formalism.

A violation of the Principle of Equivalence could arise from the possibility that the $\mathrm{K}^{0}$ and $\overline{\mathrm{K}}^{0}$ might have different interactions with the surrounding astrophysical matter distribution via background fields of tensor, vector and scalar types. Possible sources of such interactions are astrophysical bodies at generic distances $r$. One parametrizes the possible magnitudes of their effects relative to the conventional gravitational potential $U=G_{N} M / r$ due to a body of mass $M$, where $G_{N}$ is the Newton's constant, by introducing relative couplings $g$ and $\bar{g}$ for the $\mathrm{K}^{0}$ and $\overline{\mathrm{K}}^{0}$, respectively. Any matter-antimatter difference in the couplings of a field of spin $J$ and effective range $r_{J},(g-\bar{g})_{J}$, would entail an effective violation of the Principle of Equivalence, since it would violate the universality of free fall for the $\mathrm{K}^{0}$ and $\overline{\mathrm{K}}^{0}$. Such effects depend explicitly on the potential $U$ of the gravitational interaction and have an exponential dependence on the field effective range $r_{J}$.

In the case of a tensor gravitational interaction the effective $\mathrm{K}^{0}-\overline{\mathrm{K}}^{0}$ mass difference acquires the form [19]:

$\delta m_{\mathrm{eff}}=M_{\mathrm{K}^{0}}(g-\bar{g})_{2} \frac{U}{c^{2}}\left(1+v^{2} / c^{2}\right) \gamma^{2} \mathrm{e}^{-r / r_{2}}$,

where $\gamma=1 / \sqrt{1-v^{2} / c^{2}}$. In the case of a vector interaction, we assume that any vector interaction couples to the four-velocity field of the neutral kaon via $\int d t^{\prime} g_{V} V_{\mu} \dot{x}^{\mu}$, with $V_{\mu}=\left(U / c^{2}, 0,0,0\right)$ for static sources, leading to

$\delta m_{\mathrm{eff}}=M_{\mathrm{K}^{0}}(g-\bar{g})_{1} \frac{U}{c^{2}} \gamma \mathrm{e}^{-r / r_{1}}$. 
Finally, in the case of scalar interactions, we assume a "dilaton-like" coupling to the trace of the stresstensor of the kaon system, as would be the case for a Brans-Dicke scale factor, leading to

$$
\delta m_{\mathrm{eff}}=M_{\mathrm{K}^{0}}(g-\bar{g})_{0} \frac{U}{c^{2}} \mathrm{e}^{-r / r_{0}} .
$$

We emphasize the different functional forms for the tensor, vector and scalar interactions given in Eqs. (2)-(4), in particular the energy independence of the scalar interaction. Within the framework of a conventional quantum field theory we would expect that $(g-\bar{g})_{J}=0$ for $J=0$ or 2 , whereas $(g-\bar{g})_{1} \neq 0$ could be generated by graviphoton exchange [9].

Such $\mathrm{K}^{0}-\overline{\mathrm{K}}^{0}$ mass differences modify the values of the $\mathrm{CP}$-violation observables:

$\left|\eta_{+-}\right|^{2} \simeq|\epsilon|^{2}+\frac{\left(\delta m_{\text {eff }}\right)^{2}}{8(\Delta m)^{2}}$

and

$\tan \phi_{+-} \simeq \tan \phi_{\mathrm{sw}}+\frac{1}{2 \sqrt{2}} \frac{\delta m_{\mathrm{eff}}}{\Delta m|\epsilon|}\left(\tan ^{2} \phi_{\mathrm{sw}}+1\right)$,

where $\phi_{\mathrm{sw}}$ is the superweak phase [3]. Limits on $(g-\bar{g})_{J}$ for interactions of any sufficiently large range may be obtained by searching for possible modulations of $\left|\eta_{+-}\right|$and $\phi_{+-}$due to changes in the effective potential, e.g., as the Earth orbits the Sun, which would induce an annual modulation in $\phi_{+-}$ and $\left|\eta_{+-}\right|$.

From the expressions for $\delta m_{\text {eff }}$, quoted above, we can calculate the variations of $\phi_{+-}$and $\left|\eta_{+-}\right|$with the variations $\Delta U$ of the astrophysical potentials, as determined from astrophysical measurements [20]. One thus obtains the following explicit expressions for $(g-\bar{g})_{J}$

$|g-\bar{g}|_{J}=2 \sqrt{2} c^{2} \frac{\Delta m}{M_{\mathrm{K}^{0}}}\left(\frac{\Delta\left(\left|\eta_{+-}\right|^{2}\right)}{\Delta\left(U^{2}\right)}\right)^{\frac{1}{2}} \xi_{J} \mathrm{e}^{r / r_{J}}$

and

$$
\begin{aligned}
(g-\bar{g})_{J}= & 2 \sqrt{2} c^{2} \frac{\Delta m}{M_{\mathrm{K}^{0}}} \frac{|\epsilon|}{\left(1+\tan ^{2} \phi_{\mathrm{sw}}\right)} \frac{\Delta \tan \phi_{+-}}{\Delta U} \\
& \times \xi_{J} \mathrm{e}^{r / r_{J}}
\end{aligned}
$$

where $\Delta\left(\left|\eta_{+-}\right|^{2}\right)$ and $\Delta \tan \phi_{+-}$are the variations in the observable parameters associated with a variation $\Delta U$, in the gravitational potential, and $\xi_{J}=1, \gamma^{-1}$ and $\left[\gamma^{2}\left(1+v^{2} / c^{2}\right)\right]^{-1}$ are the energy dependences for interactions with $J=0,1$ and 2 . It is clear that the sign of $(g-\bar{g})_{J}$ may be determined from Eq. (8), but this is not the case for a measurement of $\left|\eta_{+-}\right|$, as can be seen from Eq. (7). On the other hand, the numerical sensitivity of (7) is somewhat greater, as we shall see later. The limits quoted in the following refer always to $|g-\bar{g}|_{J}$.

We note that Eqs. (7) and (8) depend on $\Delta m$, for which we take the world average value $\Delta m=(3.491$ $\pm 0.009) \times 10^{-12} \mathrm{MeV}$ [3]. In evaluating (8) we take $|\epsilon|=(2.295 \pm 0.083) \times 10^{-3}$, and the superweak phase $\phi_{\text {sw }}=43.49^{0} \pm 0.08^{0}$ [3]. A straightforward calculation shows that the gravitational corrections in $\Delta m, \phi_{\mathrm{sw}}$ and $|\epsilon|$ are suppressed by higher powers of $|g-\bar{g}|_{J}$. Hence their neglect is justified by the small upper limits that we find below on $|g-\bar{g}|_{J}$ from searches for modulations in $\left|\eta_{+-}\right|$and $\phi_{+-}$. We also note that the present experimental measurements of $\epsilon^{\prime}$ [21] permit us to neglect possible direct CP violation in the $\mathrm{K}^{0}\left(\overline{\mathrm{K}}^{0}\right) \rightarrow \pi^{+} \pi^{-}$decay.

\section{Experimental search for violations of the Equivalence Principle}

Our primary limits on $|g-\bar{g}|_{J}$ will be based on possible annual, monthly and diurnal modulations of $\left|\eta_{+-}\right|$and $\phi_{+-}$, associated with potentials generated by the Sun, Moon and Earth; we comment also on possible energy-dependent effects in the observables (5) and (6), which could be present for vector and tensor interactions. Finally, we give limits on $\mid g-$ $\left.\bar{g}\right|_{J}$ based on the experimental upper limit of the effective mass difference $|\delta m|$, in association with galactic and extra-galactic potentials.

The data used for this analysis comprise the full data set of 70 million $\mathrm{K}^{0}\left(\overline{\mathrm{K}}^{0}\right) \rightarrow \pi^{+} \pi^{-}$decays collected in the CPLEAR experiment in the years 1993, 1994 and 1995. The CPLEAR experiment is described in detail elsewhere [22]. The CP-violation parameters $\left|\eta_{+-}\right|$and $\phi_{+-}$were determined from the asymmetry $A_{+-}(\tau)$ formed from the measured 
numbers of $\mathrm{K}^{0}$ and $\overline{\mathrm{K}}^{0}$ decaying to $\pi^{+} \pi^{-}, N(\tau)$ and $\bar{N}(\tau)$, as functions of the decay time $\tau[4,23]$ :

$$
\begin{aligned}
A_{+-}(\tau) & =\frac{\bar{N}(\tau)-\alpha N(\tau)}{\bar{N}(\tau)+\alpha N(\tau)} \\
& =-2 \frac{\left|\eta_{+-}\right| \mathrm{e}^{\frac{1}{2}\left(\Gamma_{\mathrm{S}}-\Gamma_{\mathrm{L}}\right) \tau} \cos \left(\Delta m \tau-\phi_{+-}\right)}{1+\left|\eta_{+-}\right|^{2} \mathrm{e}^{\left(\Gamma_{\mathrm{S}}-\Gamma_{\mathrm{L}}\right) \tau}}
\end{aligned}
$$

where $\Delta m$ is the $\mathrm{K}_{\mathrm{L}}-\mathrm{K}_{\mathrm{S}}$ mass difference and $\Gamma_{\mathrm{L}}\left(\Gamma_{\mathrm{S}}\right)$ the $\mathrm{K}_{\mathrm{L}}\left(\mathrm{K}_{\mathrm{S}}\right)$ decay width, and the normalization factor $\alpha$ is defined in Ref. [23].

We have verified that the performance of the detector has been uniform for the duration of the data-taking periods. This stability was monitored by measuring the mass and width of both charged and neutral $\mathrm{K}^{*} \mathrm{~s}$, decaying into a kaon and a pion, for each of the years 1993, 1994 and 1995 separately. These quantities were found to be stable to the level of $10^{-3}$. Moreover we display in Fig. 1 the values of
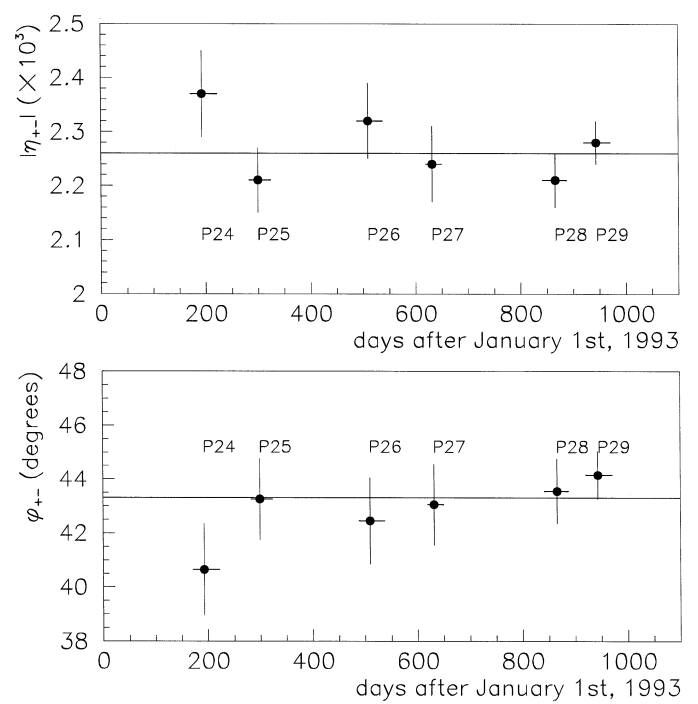

Fig. 1. The values of $\left|\eta_{+-}\right|$and $\phi_{+-}$measured during the calendar years 1993, 1994 and 1995, demonstrating the long-term stability of the detector during different running periods (P24P29). The horizontal error bars correspond to the duration of each period and the centre is its mean weighted by the number of events collected per day. For each observable, the solid line is the result of the fit with a constant and corresponds to our average value. The $\chi^{2} /$ ndf values are $4.7 / 5$ and $3.7 / 5$, respectively, for $\left|\eta_{+-}\right|$and $\phi_{+-}$. $\left|\eta_{+-}\right|$and $\phi_{+-}$found using data from different running periods during these years. We note that many effects that might cause time variations, faking a correlation in the performance of the CPLEAR detector, cancel between particles and antiparticles, which is one of the design features of CPLEAR [24].

The data are then split into subsamples corresponding to different values of the gravitational potential of the Sun, Moon and Earth. Although the systematic errors on $\left|\eta_{+-}\right|$and $\phi_{+-}$are the same for all the subsamples, for the purpose of the present study a number of checks were performed on sources of systematic errors that might induce false correlations with external variables. These include possible variations in the size and energy scales of the detector due to temperature or other effects that could mimic annual or diurnal modulations in the detector response. We conclude that all the identified systematic errors are negligible compared with the statistical errors in each of the searches itemized below.

In view of these searches, a look-up table was generated from the CPLEAR logbook data, which associate run number, date and time with each datatape written. The run number of every event is stored with it. The date and time of each run was used to produce the look-up table, which provides the gravitational potential for each run number, corrected for the position of the experiment at that time and the precession and nutation of the Earth's axis.

Values of $\left|\eta_{+-}\right|$and $\phi_{+-}$were calculated for different subsamples. Limits on $|g-\bar{g}|_{J}$ were determined from the slopes of linear fits with Eqs. (7) and (8) to the data points. All limits are given at a $95 \%$ CL. Note that in (7) only a non-negative value of $|g-\bar{g}|_{J}$ is allowed. When we get a negative central value from a fit, we use the tables in [26] to translate it to a $95 \%$ CL limit.

We have searched for the following possible effects, first giving limits for a spin-0 interaction of infinite range, later for spin- 1 and spin- 2 interactions and finally presenting a compilation in which finiterange effects are taken into account:

- A possible diurnal effect:

As a systematic check, the data were split into six separate samples according to the time of day in order to search for any variation in $\left|\eta_{+-}\right|$and $\phi_{+-}$. The measured values of $\left|\eta_{+-}\right|$and $\phi_{+-}$are plotted versus the time of day in four-hour bins in 
Fig. 2. Any astrophysical effect here is expected to be much smaller than in the other cases, and indeed none is found. We do not use these data to extract upper limits on $|g-\bar{g}|_{J}$.

- Annual modulation due to the Sun's gravitational potential:

The CPLEAR data were analyzed with respect to the time of year, in order to investigate any possible correlation with the variations $\Delta U$ in the gravitational potential of the Sun, due to the eccentricity of 0.0167 in the Earth's orbit. The total of the data was split in ten samples of roughly 7 million events each. The quantities $\left|\eta_{+-}\right|$and $\phi_{+-}$were calculated for each data sample and the mean time for the sample was obtained as a weighted mean. The Sun's distance at this time was used to find the corresponding mean gravitational potential. The results for $\left|\eta_{+-}\right|$ and $\phi_{+-}$are shown in Fig. 3 as functions of the Sun's gravitational potential. Within statistical errors, no significant correlation is seen between $\left|\eta_{+-}\right|$or $\phi_{+-}$and the potential. The slopes from the fits and their errors are used with Eq. (7) for $\left|\eta_{+-}\right|$and Eq. (8) for $\phi_{+-}$to calculate limits on $|g-\bar{g}|_{0}$ of $6.5 \times 10^{-9}$ and $1.2 \times 10^{-8}$, respectively. The fit results (one- $\sigma$ bands) are also shown in Fig. 3.
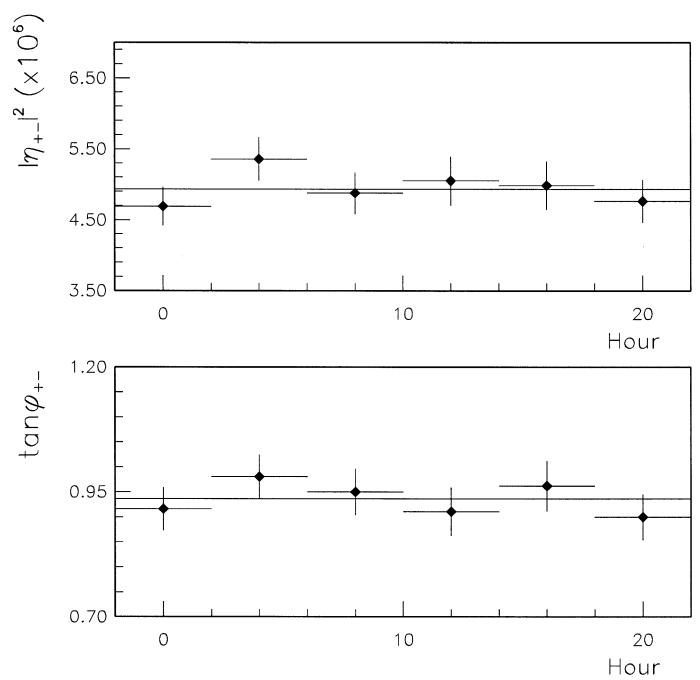

Fig. 2. Measurements of $\left|\eta_{+-}\right|^{2}$ and $\tan \phi_{+-}$as functions of the time of day. For each observable, the solid line is the result of the fit with a constant. The $\chi^{2} /$ ndf values are $3.2 / 5$ and $2.6 / 5$, respectively, for $\left|\eta_{+-}\right|^{2}$ and $\tan \phi_{+-}$.
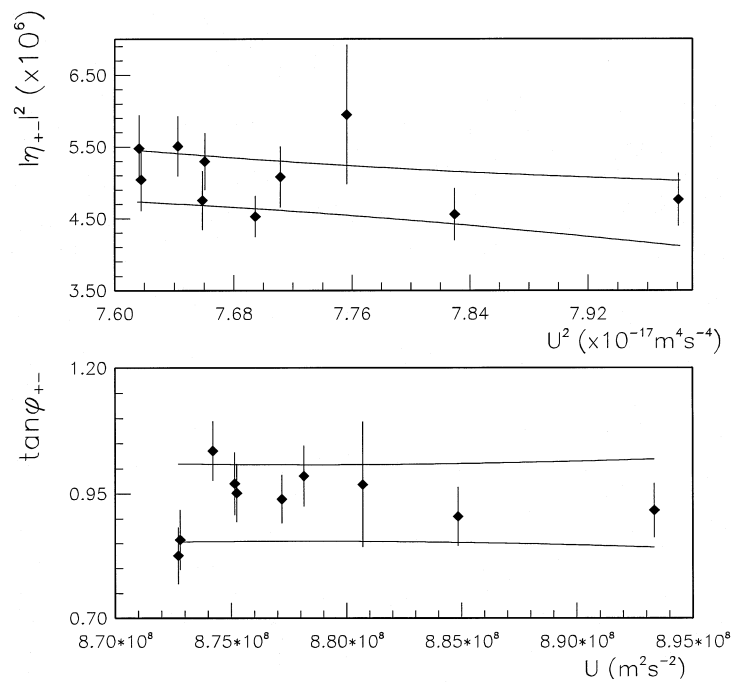

Fig. 3. Measurements of $\left|\eta_{+-}\right|^{2}$ and $\tan \phi_{+-}$as functions of the gravitational potential of the Sun. The lines represent the $\pm 1 \sigma$ limits of the region around the central values given by the fit. Note that in the top plot $\left(\left|\eta_{+-}\right|^{2}\right)$ the physical value of the slope cannot be negative, as can be seen from Eq. (7).

- Monthly modulation due to the Moon's gravitational potential:

A search was made for possible monthly variations of $\left|\eta_{+-}\right|$and $\phi_{+-}$with the changing gravitational potential due to the Moon. Results are shown in Fig. 4. No significant correlation is observed for either $\left|\eta_{+-}\right|$or $\phi_{+-}$, leading to upper limits on $|g-\bar{g}|_{0}$ of $2.0 \times 10^{-4}$ and $1.8 \times$ $10^{-4}$ from $\left|\eta_{+-}\right|$and $\phi_{+-}$, respectively.

- The Earth's gravitational potential:

The data have been split into two subsamples, for neutral kaons travelling towards or away from the earth (upwards or downwards). No significant variation in $\left|\eta_{+-}\right|$or $\phi_{+-}$is observed, leading to limits on $|g-\bar{g}|_{0}$ of $6.4 \times 10^{-5}$ and $3.7 \times 10^{-1}$ respectively. A systematic check was made by an analogous splitting of the data, but where the neutral kaon travelled horizontally, i.e., perpendicular to the Earth's gravitational field. Again, no significant variation in $\left|\eta_{+-}\right|$or $\phi_{+-}$was found.

- Galactic and extra-galactic gravitational potentials:

The time scale of the CPLEAR experiment is clearly too short to measure any variation of 

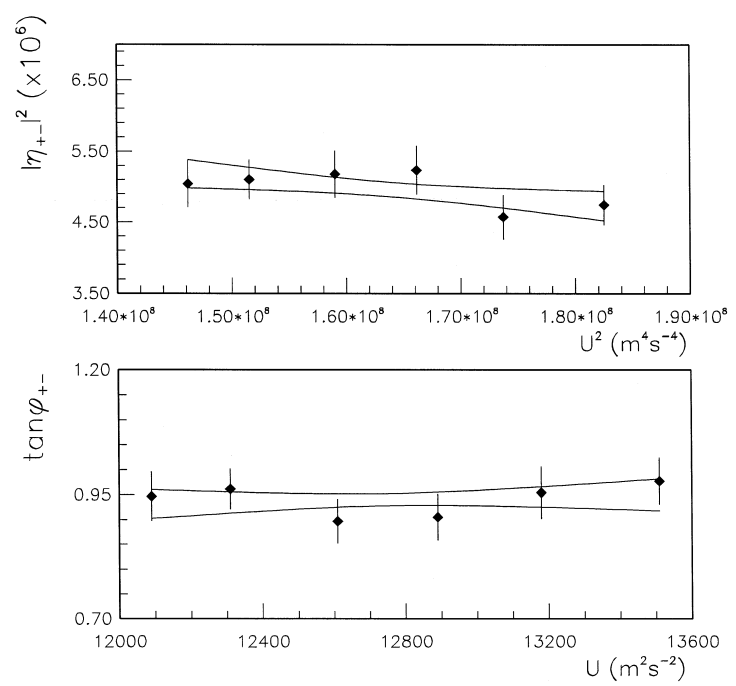

Fig. 4. Measurements of $\left|\eta_{+-}\right|^{2}$ and $\tan \phi_{+-}$as functions of the gravitational potential of the Moon and the corresponding $\pm 1 \sigma$ regions around the central values given by the fit.

$\left|\eta_{+-}\right|$or $\phi_{+-}$with the change in potential as the Earth orbits the galactic centre. However, we can place a limit on $|g-\bar{g}|$ by considering the difference between the energies of $\mathrm{K}^{0}$ and $\overline{\mathrm{K}}^{0}$ in a common effective galactocentric potential associated to a force with galactic range [20]. We take $|\delta m|=(-2.6 \pm 2.8) \times 10^{-19} \mathrm{GeV}$, as obtained by CPLEAR [4]. In this way, we find an upper limit on $|g-\bar{g}|_{0}$ of $1.4 \times 10^{-12}$ for a force with range longer than the distance of the Earth from the galactic centre, assuming conservatively a mass of $10^{11}$ solar masses for the galaxy ${ }^{1}$.

This type of limit may be extended by considering the possible effective potential generated by the Virgo cluster (or the Shapley supercluster), which is likely to be the most significant extragalactic source. In these cases the relevant masses and distances are less well known. However, these have been estimated to be $10^{14}\left(5 \times 10^{16}\right)$ solar masses and 15-20 (250) Mpc respectively [20], giving upper limits for $|g-\bar{g}|_{0}$ of $0.9 \times$ $10^{-12}\left(0.7 \times 10^{-13}\right)$. This may be compared to the similar analysis in [19].

\footnotetext{
${ }^{1}$ The corresponding limit based on the Sun potential alone is $|g-\bar{g}|_{0}<0.7 \times 10^{-10}$.
}

It should be emphasized once more that this method used for the galactic and supercluster cases requires the use of an absolute potential, and hence involves an extra theoretical assumption. It should be noted that this method cannot be applied to the Sun, Earth and Moon, since presumably the galactic and/or extragalactic potentials dominate at the Earth surface, if the range is large. The method of placing bounds using $\phi_{+-}$ and $\left|\eta_{+-}\right|$modulations avoids the use of absolute potentials altogether, and therefore the bounds so obtained are the best available from a model-independent point of view.

In all the cases considered, the values obtained for $\left|\eta_{+-}\right|$and $\phi_{+-}$do not show a significant dependence on the potential, within 1.5 standard deviations. The above limits on $|g-\bar{g}|$ refer to a spin-0 interaction. Table 1 summarizes the best limits on $|g-\bar{g}|$ obtained from $\left|\eta_{+-}\right|$and $\phi_{+-}$for tensor, vector and scalar interactions. We note that the limits from $\left|\eta_{+-}\right|$are usually better than those from the $\phi_{+-}$data. The limits for galactic and extragalactic gravitational potentials, obtained from $\delta m$, are also reported in Table 1. The limits given in Table 1 were all calculated for forces of range much longer than the corresponding astrophysical distance scale, so that the exponential factors $\exp \left(r / r_{J}\right) \simeq 1$.

It has been suggested previously [18] that all the $\mathrm{CP}$ violation observed in the neutral-kaon mass matrix might be due to the interaction with an astrophysical source. Our results, on the absence of modulations correlated, e.g., with the Earth-Sun distance do not allow us to reject this hypothesis, although they can be used to constrain the allowed couplings of conjectural particles as functions of their masses [25].

Table 1

Summary of limits on $|g-\bar{g}|$ for spin 0,1 and 2 interactions

\begin{tabular}{llll}
\hline Source & Spin 0 & Spin 1 & Spin 2 \\
\hline Earth & $6.4 \times 10^{-5}$ & $4.1 \times 10^{-5}$ & $1.7 \times 10^{-5}$ \\
Moon & $1.8 \times 10^{-4}$ & $7.4 \times 10^{-5}$ & $4.8 \times 10^{-5}$ \\
Sun & $6.5 \times 10^{-9}$ & $4.3 \times 10^{-9}$ & $1.8 \times 10^{-9}$ \\
Galaxy & $1.4 \times 10^{-12}$ & $9.1 \times 10^{-13}$ & $3.8 \times 10^{-13}$ \\
Supercluster & $7.0 \times 10^{-14}$ & $4.6 \times 10^{-14}$ & $1.9 \times 10^{-14}$ \\
\hline
\end{tabular}




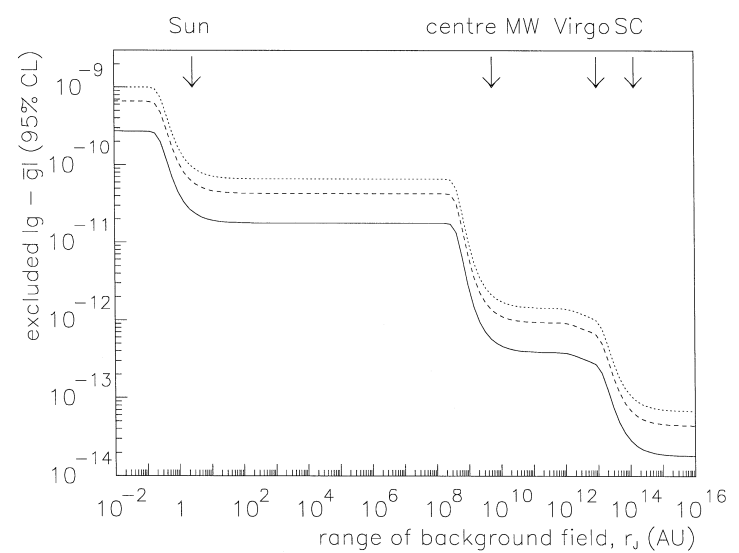

Fig. 5. Limits on $|g-\bar{g}|_{J}$ arising from the measured $\mathrm{K}^{0}-\overline{\mathrm{K}}^{0}$ mass difference [4] as a function of the effective interaction range. Labels along the top denote the distances to several astronomical bodies (Milky Way: MW, Shapley supercluster: SC) measured in Astronomical units (AU). The curves are upper limits shown separately for tensor (solid line), vector (dashed line) and scalar (dotted line) interactions.

In the cases of spin-1 and spin-2 interactions, limits can in principle also be obtained from studies of the energy dependence of parameters of the $\mathrm{K}^{0}$ $\overline{\mathrm{K}}^{0}$ system $^{2}$. We note that, in view of the small range of $\gamma$ involved in the CPLEAR experiment $(\gamma=1.54$ at the average kaon momentum) and the stringent upper limits on $|g-\bar{g}|$ obtained above, CPLEAR is not sufficiently sensitive to constrain significantly interactions with the energy dependences given in (2) and (3). However, we point out that a similar analysis of the combined data taken by CPLEAR and experiments E731, E773 at FNAL [28] would have considerably greater sensitivity to spin-1 and particularly spin- 2 interactions, since the overall range of $\gamma$ would extend up to the order of $10^{6}$.

Finally, we use the limit on the $\mathrm{K}^{0}-\overline{\mathrm{K}}^{0}$ mass difference determined by CPLEAR [4] and the full functional form of Eqs. (2)-(4) to compile the limits on $|g-\bar{g}|_{J}$ for a spin- $J$ interaction as a function of the finite range $r_{J}$. These limits are shown in Fig. 5 . It should be emphasized that the more stringent limits at large range have intrinsically larger uncer-

\footnotetext{
${ }^{2}$ These can also be used to constrain the phase difference parameters in the generalized interaction formalism of [27] for violations of the Equivalence Principle.
}

tainties, associated with uncertainties in modelling large-scale structures.

\section{Conclusion}

We have found no evidence for any variation of $\left|\eta_{+-}\right|$or $\phi_{+-}$associated with possible effective potentials generated by the Earth, Moon or Sun. We have used our data to establish stringent upper limits on possible effective spin- $0,-1$ or -2 interactions that might induce apparent deviations from the Principle of Equivalence, which we have given as functions of their possible ranges.

\section{Acknowledgements}

We would like to thank the CERN LEAR staff for their support and co-operation, as well as the technical and engineering staff of our institutes. We would also like to thank M. Currie and J. Lockley at Starlink (the computing facility for UK astronomers) for their help with Starlink's software. We thank K. Zioutas for his interest, and also thank M. Davis, A. Dekel and A. Eldar for their advice. This work was supported by the following agencies: the French CNRS/Institut National de Physique Nucléaire et de Physique des Particules (IN2P3), the French Commissariat à l'Energie Atomique (CEA), the Greek General Secretariat of Research and Technology, the Netherlands Foundation for Fundamental Research on Matter (FOM), the Portuguese JNICT, the Ministry of Science and Technology of the Republic of Slovenia, the Swedish Natural Science Research Council, the Swiss National Science Foundation, the UK Particle Physics and Astronomy Research Council (PPARC), the US National Science Foundation (NSF) and the US Department of Energy (DOE).

\section{References}

[1] J.S. Bell, Proc. Royal Soc. A 231 (1955) 479; W. Pauli, in: W. Pauli, L. Rosenfeld, V. Weisskopf (Eds.), Niels Bohr and the Development of Physics, McGraw Hill, New York, 1955; G. Lüders, Ann. Phys. (NY) 2 (1957) 1; R. Jost, Helv. Phys. Acta 30 (1957) 409. 
[2] N. Tanner, R. Dalitz, Ann. Phys. (NY) 171 (1986) 463; C.D. Buchanan et al., Phys. Rev. D 45 (1992) 4088; C.O. Dib, R.D. Peccei, Phys. Rev. D 46 (1992) 2265.

[3] Particle Data Group, R.M. Barnett, Phys. Rev. D 54 (1996) 412.

[4] CPLEAR Collaboration, Results on CP, T, CPT symmetries with tagged $\mathrm{K}^{0}$ and $\overline{\mathrm{K}}^{0}$ by CPLEAR, Proc. ICHEP'98, Vancouver, 1998, and papers to be published.

[5] J. Ellis, J. Hagelin, D.V. Nanopoulos, M. Srednicki, Nucl. Phys. B 241 (1984) 381.

[6] J. Ellis, N.E. Mavromatos, D.V. Nanopoulos, Phys. Lett. B 293 (1992) 142; Int. J. Mod. Phys. A 11 (1996) 1489; J. Ellis, J. Lopez, N.E. Mavromatos, D.V. Nanopoulos, Phys. Rev. D 53 (1996) 3846; P. Huet, M.E. Peskin, Nucl. Phys. B 434 (1995) 3.

[7] R. Adler et al., CPLEAR Collaboration, J. Ellis, J. Lopez, N.E. Mavromatos, D.V. Nanopoulos, Phys. Lett. B 364 (1995) 239.

[8] R. Wald, Phys. Rev. D 21 (1980) 2742; D. Page, Gen. Rel. Grav. 14 (1982) 299.

[9] J. Scherk, Phys. Lett. B 88 (1979) 265; see also: S. Belucci, V. Faraoni, Phys. Rev. D 49 (1994) 2922; Phys. Lett. B 377 (1996) 55, and references therein.

[10] M.L. Good, Phys. Rev. 121 (1961) 311.

[11] Y. Su, Phys. Rev. D 50 (1994) 3614.

[12] J.G. Williams, Phys. Rev. D 53 (1996) 6730.

[13] R.F.C. Vessot, Phys. Rev. Lett. 45 (1980) 2081.

[14] T.E. Chupp et al., Phys. Rev. Lett. 63 (1989) 1541; S.K. Lamoreaux et al., Phys. Rev. Lett. 57 (1986) 3125; J.D. Prestage et al., Phys. Rev. Lett. 54 (1985) 2387.

[15] J.D. Prestage, Phys. Rev. Lett. 74 (1995) 3511.
[16] G. Gabrielse, Phys. Rev. Lett. 65 (1990) 1317.

[17] R.J. Hughes, M.H. Holzscheiter, Phys. Rev. Lett. 66 (1991) 854.

[18] J.S. Bell, in: P. Bloch, P. Pavlopoulos, R. Klapisch (Eds.) Fundamental Symmetries, Plenum, New York, 1987; for an alternative formulation, see G. Chardin, J.-M. Rax, Phys. Lett. B 282 (1992) 256; G. Chardin, Nucl. Phys. A 558 (1993) 477c; Hyp. Int. 109 (1997) 83.

[19] R.J. Hughes, Phys. Rev. D 46 (1992) R2283.

[20] M. Davis, M.A. Strauss, A. Yahil, Ap. J. 372 (1991) 394; M. Davis, A. Dekel, A. Eldar, private communication.

[21] P. Buchholz et al., Phys. Lett. B 317 (1993) 233; A.P. Barker et al., Phys. Rev. Lett. 70 (1993) 1203.

[22] CPLEAR Collaboration, R. Adler, Nucl. Instr. \&Meth. A 379 (1996) 76.

[23] CPLEAR Collaboration, R. Adler, Phys. Lett. B 363 (1995) 243.

[24] E. Gabathuler, P. Pavlopoulos, in: U. Gastaldi, R. Klapisch (Eds.), Strong and Weak CP Violation at LEAR, Proc. Workshop on Physics at LEAR with Low Energy Cooled Antiprotons, Erice, 1982, Plenum, New York, 1984, p. 747.

[25] M.N. Nieto, T. Goldman, Phys. Rep. 5 (1991) 221; R.J. Hughes, T. Goldman, M.N. Nieto, in: P. Bloch, P. Pavlopoulos, R. Klapisch (Eds.), Fundamental Symmetries, Plenum, New York, 1987.

[26] G.J. Feldman, R.D. Cousins, Phys. Rev. D 57 (1998) 3873.

[27] T. Hambye, R.B. Mann, U. Sarkar, hep-ph/9608423; Phys. Lett. B 421 (1998) 105; Phys. Rev. 58 (1998) 025003.

[28] L.K. Gibbons et al., Phys. Rev. Lett. 70 (1993) 1199; B. Schwingenheuer et al., Phys. Rev. Lett. 74 (1995) 4376. 